\title{
Oblique parameters and extra generations via OPUCEM
}

\author{
Ece Aşılar $^{1, a}$, Esin Çavlan ${ }^{2, b}$, Oktay Doğangün ${ }^{3, c}$, Sinan Kefeli ${ }^{4, d}$, V. Erkcan Özcan ${ }^{4, e}$, Mehmet Şahin $^{5, f}$, \\ Gökhan Ünel ${ }^{6, g}$ \\ ${ }^{1}$ Physics Department, Middle East Technical University, Ankara, Turkey \\ ${ }^{2}$ Physics Department, Afyon Kocatepe University, Afyon, Turkey \\ ${ }^{3}$ Department of Physical Sciences, University of Naples \& INFN, Naples, Italy \\ ${ }^{4}$ Physics Department, Boğaziçi University, Bebek, Istanbul, Turkey \\ ${ }^{5}$ Department of Physics, Usak University, Usak, Turkey \\ ${ }^{6}$ Department of Physics and Astronomy, University of California at Irvine, Irvine, USA
}

Received: 23 January 2012 / Revised: 7 March 2012 / Published online: 11 April 2012

(C) The Author(s) 2012. This article is published with open access at Springerlink.com

\begin{abstract}
Recent improvements to OPUCEM, the tool for calculation of the contributions of various models to oblique parameters, are presented. OPUCEM is used to calculate the available parameter space for the four family Standard Model given the current electroweak precision data. It is shown that even with the restrictions on Higgs boson and new quark masses presented in the 2011 Autumn conferences, there is still enough space to allow a fourth generation with Dirac type neutrinos. For Majorana type neutrinos, the allowed parameter space is even larger. The electroweak precision data also appear to favor non-zero mixing between light and fourth generations, thus effectively reducing the current experimental limits on the masses of the new quarks, which assume that the mixing with the third generation is dominant. Additionally, disregarding the lack of a clear Higgs signal from the LHC and focusing only an electroweak precision data comptability, calculations with OPUCEM show that, the existing electroweak data are compatible with the presence of a 5th and also a 6th generation in certain regions of the parameter space.
\end{abstract}

\section{Introduction}

The categorization of the electroweak (EW) corrections based on their contribution types dates back to a study of

\footnotetext{
a e-mail: ece.asilar@cern.ch

be-mail: echavlan@gmail.com

c e-mail: oktay.dogangun @ cern.ch

d e-mail: sinan.kefeli@boun.edu.tr

e e-mail: Erkcan.Ozcan@cern.ch

f e-mail: mehmet.sahin@usak.edu.tr

g e-mail: Gokhan.Unel@cern.ch
}

photon propagated four-fermion processes [1]. In the original nomenclature, the corrections to vertices, box diagrams and bremsstrahlung diagrams were all considered as " $d i$ rect" whereas the propagator corrections due to vacuum polarization effects were all named as "oblique" since these participate to the computations in an indirect manner. An extended review of this approach and its application of the methodology to Beyond the Standard Model (BSM) theories helped coining the term oblique parameters [2] usually represented by the letters $S, T$ and $U$. The Standard Model is defined by the values $S=T=U=0$ for a given top quark and Higgs boson mass. In a BSM theory with new fermion doublets, the $S$ parameter estimates the size of the additional fermion sector and the $T$ parameter measures the isospin symmetry violation in that sector. In such a model, the $U$ parameter is dependent on $\mathrm{W}$ boson width, thus insensitive to new physics.

As the one-loop exact calculations for a model are tedious and error prone, an open-source $\mathrm{C} / \mathrm{C}++$ library called $\mathrm{OP}$ UCEM (Oblique Parameters Using $\mathrm{C}$ with Error-checking Machinery) was introduced to improve the reliability and reproducibility of computations in scientific publications [3]. This library provides functions to calculate the contributions to the oblique parameters from a number of BSM models. A command line program constitutes an example on how to use the library functions and a graphical user interface facilitates the library's use by non-programmers. Additionally, tools for plotting error ellipses in the $S-T$ plane and extensive documentation are provided. Among the physics models currently implemented, one can cite Standard Model with four fermion families (SM4) [4] and 2 Higgs Doublet Models (2HDM). Formulas for computing the effect of adding new fermion doublets with Majorana-type neutrinos and of the mixing between the quark generations are also 
available. The oblique parameters $S, T$ and $U$ are computed by using both exact one-loop calculations and with some well-defined approximations for various models, providing an additional machinery for error checking, beyond the various internal self-crosscheck mechanisms.

Various studies of the SM4 mass and mixing parameters performed using OPUCEM were previously discussed in [5]. This note describes the recent additions to the library, and to the command line and graphical tools, as present in the OPUCEM version 00-00-07. The overall available parameter space in the SM4 model and the compatibilities of BSM theories with further additional generations are discussed. Despite the recent negative results from the Large Hadron Collider (LHC) in the search for heavy quarks using about $1 \mathrm{fb}^{-1}$ of data [6], it is shown that there is still available room in the SM4 parameter space. The improvements to the OPUCEM package since the last publication [5], can be summarized as follows: (i) the addition of the mixing in the quark sector between the new heavy quarks and the second generation quarks, namely the function calls to calculate $T$ value in the presence of the parameter in addition to the previously available $\theta_{34}$; (ii) machinery to perform parameter scans through the command line interface, in order to facilitate the exploration of the parameter space defined by the fermion and Higgs masses and the quark mixings; (iii) extension of the graphical user interface to include the fifth and sixth generation fermion masses for both Dirac and Majorana type neutrinos. In the current implementation, if the masses of the $N$ th generation fermions are set to zero, the calculations are automatically reduced to those for a model of $N-1$ generations.

\section{OPUCEM on the SM4}

OPUCEM and Gfitter are the two open-source tools available for the calculation of the oblique parameters for a number of models ${ }^{1}$ [7]. In addition to SM4, Gfitter's theory repository includes other models such as Extra Dimensions and Little Higgs Models. However for the SM4 model, Gfitter does not incorporate the effect of the mixing between the quarks of the fourth and other generations, and it is limited only to Dirac type neutrinos. While these two tools have been created for different purposes, the fact that two recent implementations of the $S, T$ formulas exist provides an opportunity to check their reliability. As the original goal of the OPUCEM library has been to provide well-tested and errorfree results (as tested multiple times before), a comparison between the results of Gfitter and OPUCEM has been performed. For such a comparison, the input reference values

\footnotetext{
${ }^{1}$ After the first version of this paper appeared on the arXiv, the authors were informed of the G4LHC package [8] that includes the software for the SM4 $S, T$ calculations that were performed in [9].
}

and electroweak fit results should be the same in both packages. Since the Gfitter results are presented with two different sets of input values (with $U=0$ forced and $U=$ free), the reference values and the definitions of the error ellipses for the two programs are given in Table 1 for the readers convenience. The default values in OPUCEM have been obtained from the LEP Electroweak Working Group through private communication (summer 2009 results) and from the Tevatron Electroweak Working Group [10]. Figure 1 shows a scan of the fourth generation parameter space performed with OPUCEM using the Gfitter reference values for the $U=$ free case. This figure can directly be compared to the Gfitter fourth generation parameter scan results as presented in [7], as it has been prepared with the same scan and axis ranges for the new fermion and Higgs boson masses (as indicated on the figure itself), and with only Dirac-type neutrinos and no mixings between generations. The green area is the region of the $S, T$ plane that is accessible by SM4, as the input parameters are scanned through. Red and black ellipses are the $68 \%$ and $95 \%$ CL contours of the experimentally allowed $S, T$ values. The plus sign shows the SM reference point where all the oblique parameters vanish, whereas the blue horizontal and vertical lines define the center of the error ellipses given in the first row of Table 1. Comparison of this plot with the Fig. 12 of [7] shows perfect agreement between the results of the two software packages.

For the rest of this section, the Standard Model studies with four generations of fermions (SM4) is discussed so as to determine the effect of the EW precision data on $S-T$ parameter space. Cases with Dirac and Majorana type neutrinos, both stable and unstable, are considered. The aim is to perform a parameter space scan to investigate the viability of the SM4 model, given the current status of the searches and the electroweak precision data results. The initial values of mass parameters originate from the direct searches, usu-

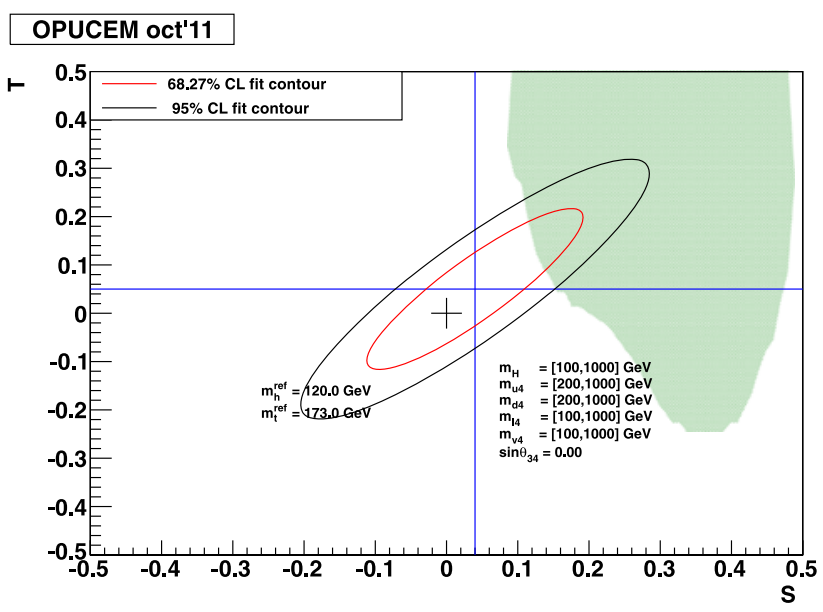

Fig. 1 A parameter scan of the SM4 model with OPUCEM using Gfitter default values and parameters. The plot has been produced in a format that allows direct comparison with [7] 
Table 1 Reference values to use for comparison between Gfitter and OPUCEM results. $\rho$ indicates the correlation coefficient between the $S$ and $T$ values

\begin{tabular}{lllllll}
\hline & $m_{H}$ & $m_{\text {top }}$ & $S$ & $T$ & $U$ & $\rho$ \\
\hline Gfitter 1 & 120 & 173 & $0.04 \pm 0.10$ & $0.05 \pm 0.11$ & $0.08 \pm 0.11$ & 0.89 \\
Gfitter 2 & 120 & 173 & $0.07 \pm 0.09$ & $0.10 \pm 0.08$ & 0 & 0.88 \\
OPUCEM & 115 & 173.1 & $0.07 \pm 0.10$ & $0.1067 \pm 0.09$ & 0 & 0.85 \\
\hline
\end{tabular}

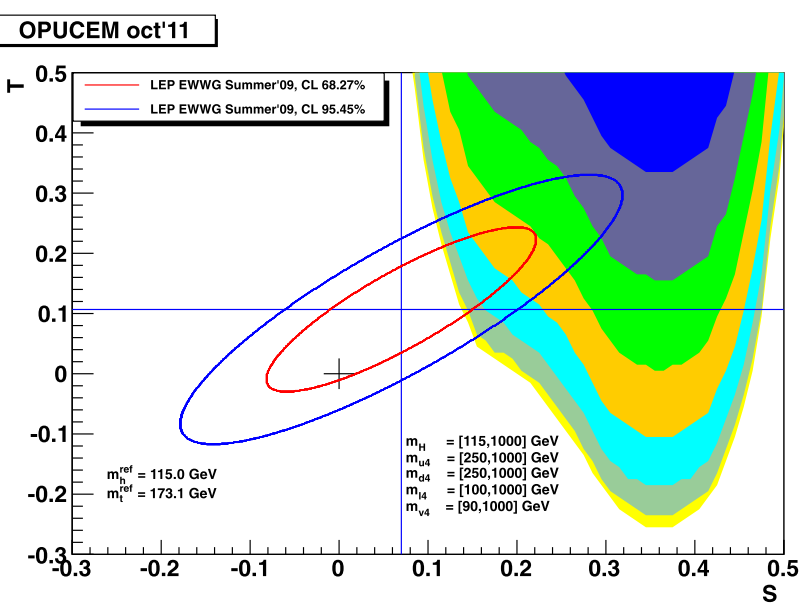

Fig. 2 Impact of the mixing between third and fourth family quarks on the fourth generation $S, T$ space for unstable (left) and stable (right) Dirac-type neutrinos obtained using the PDG mass limit values. The color-filled regions show the set of points in the plane that is reachable

ally summarized by the Particle Data Group (PDG) [11] and updated with publications from current direct search experiments. For the final values a common mass limit of $1 \mathrm{TeV}$, not too far from the partial wave unitarity bound, seems to be preferred choice in the literature. Unless stated otherwise, the step size in the mass scans of the $S-T$ plane is taken to be $10 \mathrm{GeV}$ which is a good compromise between the execution speed and the accuracy of the results. For the remainder of this work, OPUCEM defaults shown in the last row of Table 1 are used in $S-T$ parameter space plots.

\subsection{With Dirac type neutrinos}

For a fourth generation with a Dirac type neutrino, 6 parameters are commonly considered: masses of the new fermions, the Higgs boson mass and the sine of the mixing angle between the third and fourth generation quarks. The mixing between third and fourth generation quarks, $\theta_{34}$, plays an important role in the determination of the best fit to the EW precision data. Left-hand side of Fig. 2 shows the impact of this mixing on the allowed parameter space when the PDG mass limits on the new fermions mass values are considered for an unstable Dirac neutrino. In this scenario, the initial default mass pattern is set as: $m_{u 4}=m_{d 4}=250 \mathrm{GeV}$, $m_{H}=115 \mathrm{GeV}, m_{\ell 4}=100 \mathrm{GeV}$ and $m_{v 4}=90 \mathrm{GeV}$. Seven different mixing angle values are considered to investigate

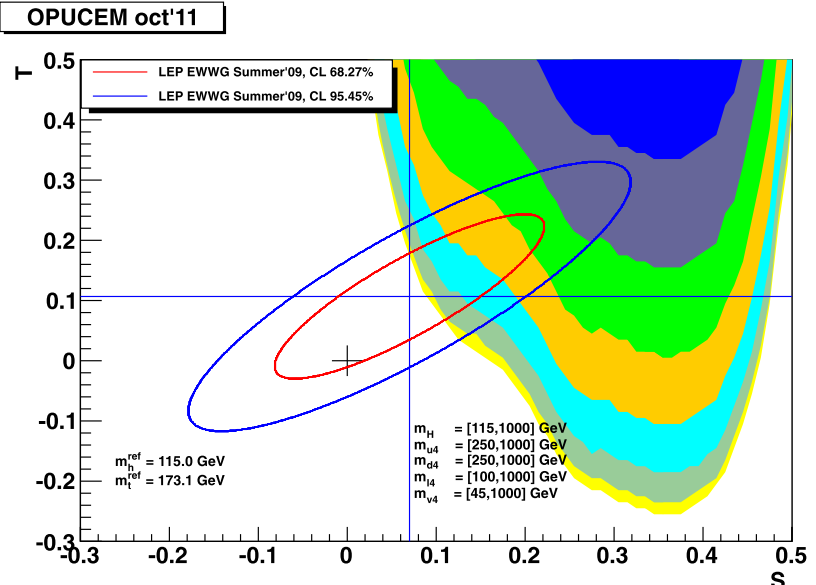

by varying all the fourth generation parameters except the mixing angles. Colors correspond to $\left|\sin \theta_{34}\right|$ varying between 0.0 and 0.6 (Color figure online)

the effect on the $S-T$ plane: $\left|\sin \theta_{34}\right|=0.0,0.1,0.2,0.3$, $0.4,0.5,0.6$. In Fig. 2 , each scan with a different mixing angle is shown as a contour with a different color, the largest contour (hence allowed parameter space) in the $S-T$ plane corresponds to no-mixing and the smallest contour, shown with dark blue, corresponds to $\left|\sin \theta_{34}\right|=0.6$. As seen from the intersection of these colored contours with the $S, T$ ellipses extracted from the EW precision data, the size of the allowed region gets smaller with increasing mixing, such that in order to be compatible with the EW precision data at the $2 \sigma$ level, the mixing between the third and fourth generation quarks has to have an upper limit, $\left|\sin \theta_{34}\right| \lesssim 0.6$, independent of the other parameters. In general, small mixing angles are preferred and so as to keep the fourth generation in the $1 \sigma$ error ellipse, the mixing angle should satisfy $\left|\sin \theta_{34}\right|<0.4$.

Since the minimum experimentally allowed mass for a stable neutrino differs from the unstable case, the parameter space is re-scanned for the stable Dirac case. As seen on the right-hand side of Fig. 2, the decrease in the neutrino mass limit downs to $45 \mathrm{GeV}$ reflects itself as an expansion of the allowed parameter space with respect to the previous case. However, the constraints for the mixing angle remain as before.

In Autumn 2011, the CMS Collaboration reported new limits on the minimum allowed heavy quark mass values 


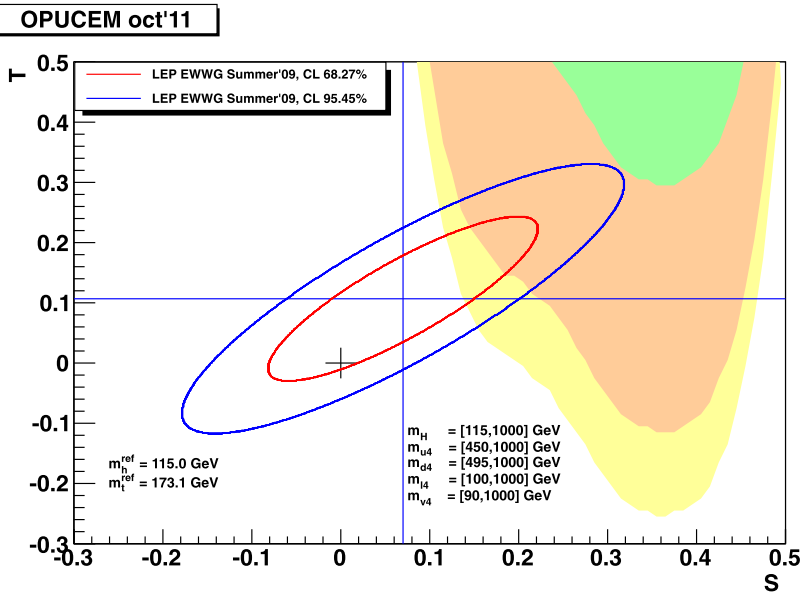

Fig. 3 Mixing angle dependence of the fourth generation quarks. $S$, $T$ parameter space for unstable (left) and stable (right) Dirac-type neutrinos using the updated mass limit values from the LHC: $m_{u 4}>$

corresponding to $m_{u 4}>450 \mathrm{GeV}$ and $m_{d 4}>495 \mathrm{GeV}$ at $95 \%$ CL [12-14]. The parameter space after this update is shown in Fig. 3. The colored contours correspond to three different mixing angle values $\left|\sin \theta_{34}\right|=0.0,0.1$ and 0.2 . In this scenario, due to the reduction of the allowed parameter space, the $2 \sigma$ error ellipse corresponds to a maximum value of the mixing angle, $\left|\sin \theta_{34}\right|<0.2$, both for stable and unstable neutrinos.

The impact of assuming a stable neutrino is better illustrated in Fig. 4 where three different mixing angle values for stable and unstable neutrinos are investigated. The considered mixing angle values are $\left|\sin \theta_{34}\right|=0.0,0.1$ and 0.2 where the allowed regions size decreases as the mixing angle increases. In the plot, darker colors represent the unstable Dirac neutrino case and light colors represent the stable case. One can see from the figure that the stable Dirac type

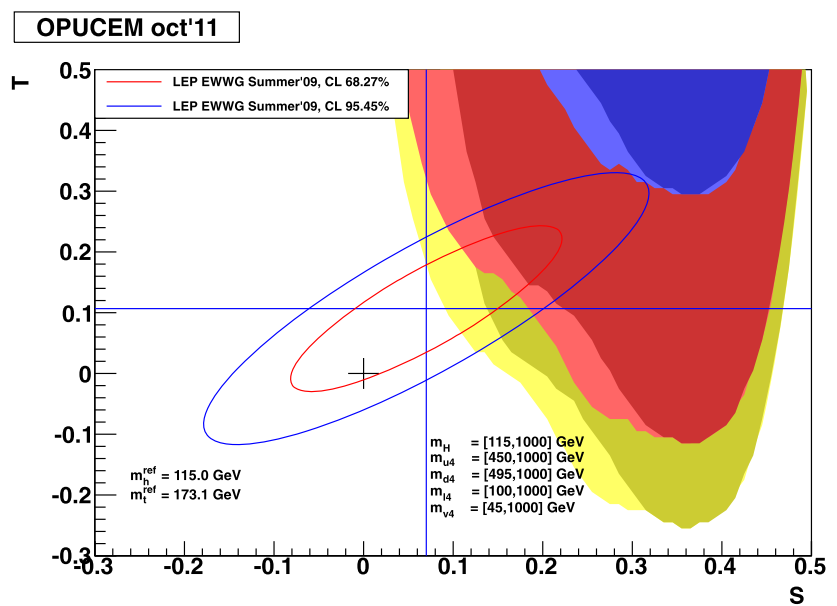

Fig. 4 Mixing angle dependence of stable vs unstable neutrinos with updated mass limit values: $m_{u 4}>450 \mathrm{GeV}$ and $m_{d 4}>495 \mathrm{GeV}$

\section{OPUCEM oct'11}

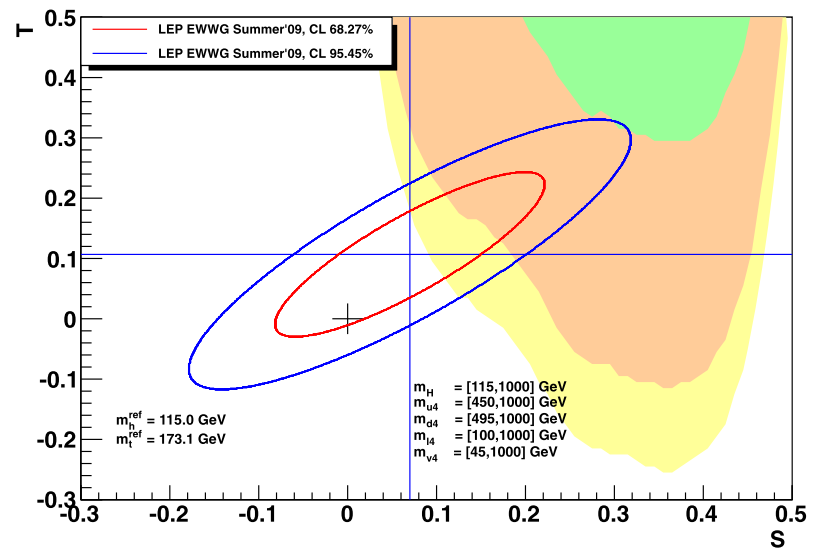

$450 \mathrm{GeV}$ and $m_{d 4}>495 \mathrm{GeV}$. The 3 colored regions correspond to $\left|\sin \theta_{34}\right|=0.0,0.1$ and 0.2 (yellow, orange and green, respectively) (Color figure online)

neutrino slightly enlarges the available parameter space in the negative $S$ direction, independently of the mixing angle.

Results from Summer 2011 which references the CMS outcomes have also excluded a SM Higgs boson in the mass range $120<m_{H}<600 \mathrm{GeV}$ with $95 \% \mathrm{CL}[15,16]$. The remaining mass windows for the Higgs boson are for a heavy one of mass larger than $600 \mathrm{GeV}$ and a light one between $115 \mathrm{GeV}$ and $120 \mathrm{GeV}$. Figure 5 indicates these states for the Dirac stable neutrino case with $\left|\sin \theta_{34}\right|=0.0$. The light (heavy) Higgs boson scenario is shown with light blue (orange). One can see that light Higgs boson is favored over a heavy one, but even a heavy Higgs boson is still compatible with a fourth generation according to the EW precision data.

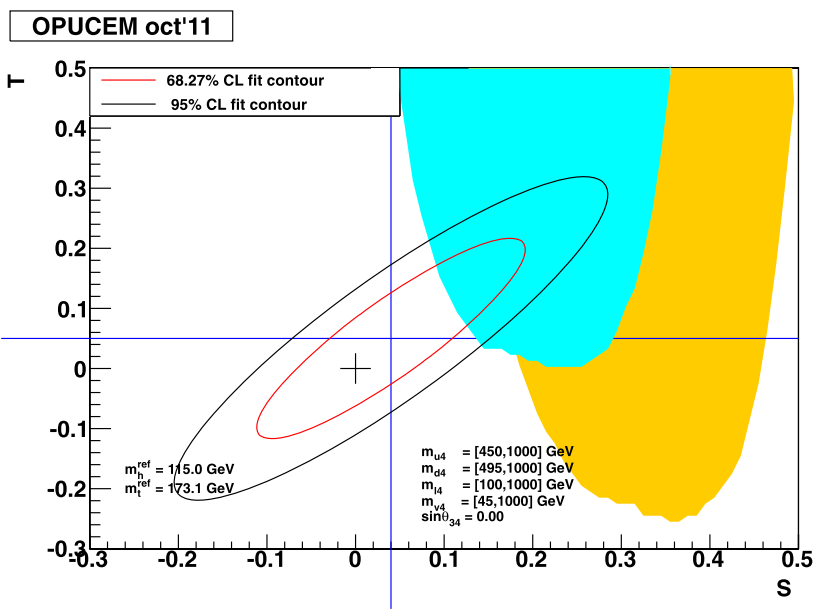

Fig. 5 Light Higgs mass (light blue) and heavy Higgs mass (orange) dependence of the fourth family fermions in stable Dirac neutrino case with updated mass limit values: $m_{u 4}>450 \mathrm{GeV}$ and $m_{d 4}>495 \mathrm{GeV}$ (Color figure online) 


\subsection{With Majorana type neutrinos}

A fourth generation with a Majorana type neutrino requires an extra mass parameter, namely the mass of the heavier neutrino denoted by $m_{N 4}$. Therefore, seven parameters have been considered for the oblique parameter calculations: masses of the new fermions including the mass of the heavier neutrino, the Higgs boson mass and the sine of the mixing angle between third and fourth generation quarks. Figure 6 contains a parameter scan for the unstable neutrino case, considering both light (left) and heavy (right) Higgs boson cases. The initial fermion mass pattern is set as: $m_{u 4}=450 \mathrm{GeV}, m_{d 4}=495 \mathrm{GeV}, m_{H}=115 \mathrm{GeV}$, $m_{\ell 4}=100 \mathrm{GeV}, m_{N 4}=1000 \mathrm{GeV}$ and $m_{v 4}=80 \mathrm{GeV}$, where the quark mass lower limits are mostly from the CMS results $[13,14,17]$. The maximum values of the masses have been taken as $1 \mathrm{TeV}$ for all fermions except for the heavier neutrino where a maximum of $4 \mathrm{TeV}$ has been considered. The light Higgs mass is tested for two values, $115 \mathrm{GeV}$ and $120 \mathrm{GeV}$, and the heavy Higgs mass is scanned from $600 \mathrm{GeV}$ to $990 \mathrm{GeV}$ in steps of $30 \mathrm{GeV}$. The mixing angle values under consideration are $\left|\sin \theta_{34}\right|=0.0,0.2,0.4$ and 0.6 represented by contours of different colors: yellow, cyan, orange and green respectively. A simple comparison of the two plots reveals that the light Higgs boson is still the preferred scenario, just like it was the case for the Dirac type neutrinos. In this scenario, although smaller mixing angles are preferred, the highest considered value of $\left|\sin \theta_{34}\right|=0.6$ can still be tuned to appear in the $1 \sigma$ error ellipse. On the other hand, for the heavy Higgs bosons case, which is less favored, the highest value of the mixing angle allowed by the $1 \sigma$ error ellipse is $\left|\sin \theta_{34}\right|=0.2$. It can also be concluded that for the Majorana type neutrinos, the additional parameter $\left(m_{N 4}\right)$ gives more flexibility to the choice of parame-

\section{OPUCEM oct'11}

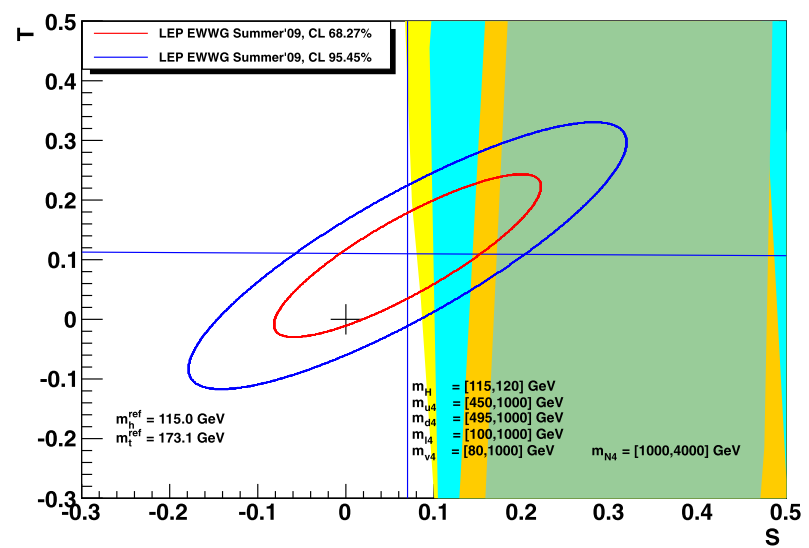

Fig. 6 Light (left) and heavy (right) Higgs mass dependence for the third and fourth family fermion in unstable Majorana case in terms of different mixing angle values. The colored regions correspond to ters. Therefore Majorana neutrino case provides a wider allowed parameter region than Dirac neutrino case. Especially by tuning the ratio of the masses of the new neutrinos, it is possible to make some previously unaccessible portions of the parameter space, compatible with the EW precision data. As a final remark, stable Majorana case was also separately considered but not included in this section. As the only difference between the two cases is the decrease of the neutrino mass lower limit down to $40 \mathrm{GeV}$, all conclusions for the unstable neutrino scenario remain also valid for the stable neutrino case, with an increase of the allowed parameter space size.

\subsection{Fourth Generation mixing with light generations}

In the literature most experimental results are reported assuming that the fourth generation quarks mix dominantly with the third generation, i.e. $B F\left(u_{4} \rightarrow W b\right)=100 \%$ and likewise for the $d_{4}$. This choice is partially motivated by the gains of using $b$-tagging in the data analysis. It is clear that a proper interpretation of the direct quark search limits requires consideration of the mixings with light generations. Furthermore, as has recently been shown in [18], taking quark mixings into account is extremely important in analyzing the EW precision data as it can significantly change the favored mass space.

OPUCEM has been used to test the implications of the quarks mixings in the case of fully degenerate fourth generation fermions, which is claimed to have been ruled out by the EW data according to the PDG [11]. As seen in Fig. 7, while the fully degenerate case without mixings is indeed ruled out with more than $99.99 \% \mathrm{CL}$, it is possible to reach better than $1 \sigma$ agreement with $\mathrm{EW}$ precision data for certain choices of the mixing parameters (in agreement with the

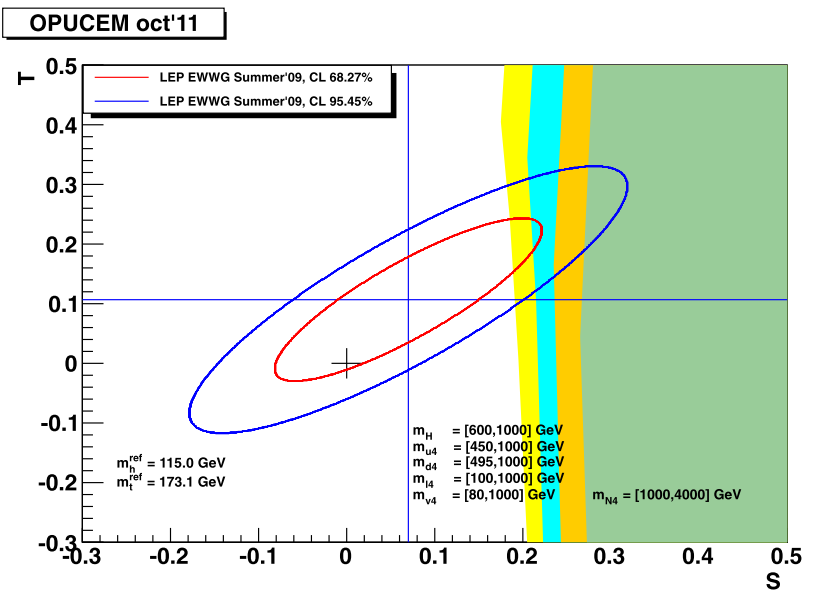

$|\sin \theta|=0.0,0.2,0.4$ and 0.6. (Different colored contours represent the allowed parameter space are yellow, cyan, orange and green respectively) (Color figure online) 


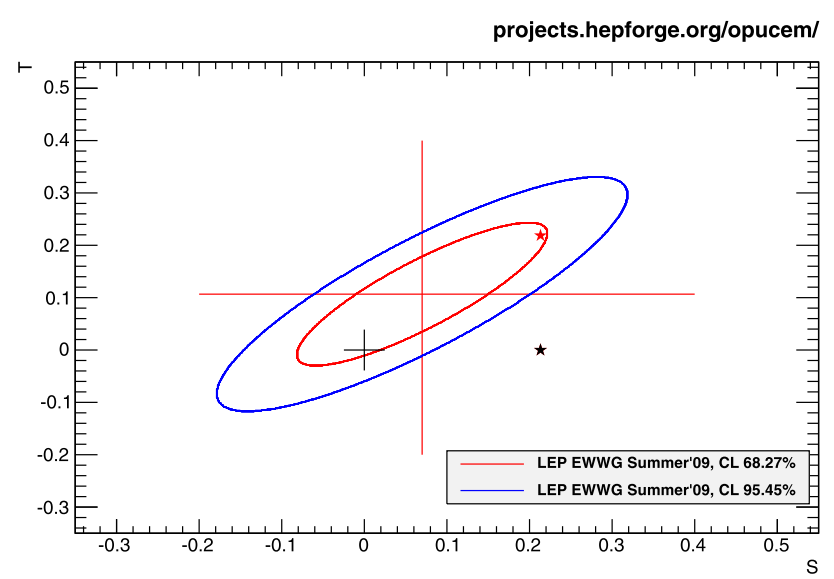

Fig. $7 S-T$ plane is drawn using OPUCEM for the fully degenerate case with the masses of all the fourth generation fermions set to $400 \mathrm{GeV}$. While with no mixing such a scenario is essentially ruled out $\left(\Delta \chi^{2}=22.1\right.$, black point $)$, with mixings of $\left|\sin \theta_{24}\right|=0.07$, $\left|\sin \theta_{34}\right|=0.14$, the $\Delta \chi^{2}=2.0$ (red point), which is within $1 \sigma$ error ellipse (Color figure online)

findings of [18]). One should note that since heavy Higgs boson masses tend to increase the $S$ parameter while decreasing $T$, the introduction of the mixings, which increase the $T$ parameter alone, can open up a significant portion of the parameter space in general.

Therefore it is worth repeating some of the parameter scans detailed in the earlier sections taking into account the mixings with the light generations. The goal chosen for this section however, is to provide some input to the interpretation of the LHC quark search limits, by setting the mass parameters to certain example values and exploring the favored values of the mixing parameters. To this end, the values of the mixings allowed by the current measurements of the $3 \times 3$ CKM matrix elements have been determined. It is worth noting that a full treatment of $4 \times 4$ CKM with EW constraints is important for determining the overall allowed parameter space, as discussed in [18]. However, to perform first order estimations, $3 \times 3 \mathrm{CKM}$ matrix measurements from the PDG [11], and imposing the unitarity condition of the $4 \times 4 \mathrm{CKM}$ matrix was sufficient to determine the values of $K_{i 4} \equiv\left|V_{i 4}\right|^{2}, i=u, c, t$. Uncertainties on $K_{i 4}$, assumed to be Gaussian, can be obtained by simple error propagation. This procedure yields: $K_{14}=0.0001 \pm 0.0006, K_{24}=-0.10 \pm 0.07, K_{34}=0.22 \pm$ 0.12 .

Noting that the allowed space for the magnitude of $K_{14}$ is much smaller than the others, the focus should be on the 2-4 and 3-4 mixings. A large number $\left(\mathcal{O}\left(10^{8}\right)\right)$ of Gaussiandistributed random $\left(K_{24}, K_{34}\right)$ pairs were generated using the center values and uncertainties computed above. For each generated non-negative $K_{24}$ and $K_{34}, S, T$ and $\Delta \chi^{2}$ from the center of the $S-T$ ellipse were computed, for a Higgs mass of $115 \mathrm{GeV}$ and assuming the neutrinos to be of Dirac type. Then using $\chi^{2}$ probability as weights, a two dimensional histogram was filled with $\left|\sin \theta_{24}\right|$ and $\left|\sin \theta_{34}\right|$ values. This procedure was repeated for a number of different fourth-generation fermion masses $\left(m_{4 \mathrm{G}}\right)$ to obtain plots of the mixing space as shown in Fig. 8.

By searching for the peak in these 2D histograms, the most favored $\left(\left|\sin \theta_{24}\right|,\left|\sin \theta_{34}\right|\right)$ pair is determined as a function of $m_{4 \mathrm{G}}$. One can observe that the favored $\theta_{24}$ decreases slightly as $m_{4 \mathrm{G}}$ increases, whereas $\theta_{34}$ shows a much more rapid decrease. It is important to note that even at low masses, favored $\theta_{24}$ is not negligible compared to $\theta_{34}$ as shown in Fig. 9. It is also worth mentioning that the favored values are not far from the results of the preliminary analysis presented in [19], despite relying on much more limited experimental data. Since the branching fraction for the decays of the fourth generation quarks into final states involving third generation is approximately given by $B F \sim\left|V_{t b^{\prime}}\right|^{2}\left(\left|V_{t b^{\prime}}\right|^{2}+\left|V_{c b^{\prime}}\right|^{2}\right)$, it is possible to extract the favored value of the $B F$ as well. As seen in Fig. 10 left plot, this value varies between $90 \%$ and $60 \%$ as a monotonicallydecreasing function of $m_{4 \mathrm{G}}$.

An extensive analysis of the scenario with non-degenerate fourth generation fermion masses requires the scan of a large parameter space. Therefore, in order to gain some insight, a particular benchmark case is considered in which a light Higgs boson decays into a pair of fourth generation neutrinos, avoiding the current Higgs search limits from the LHC experiments [20,21]. The example benchmark point is taken to be: $m_{H}=130 \mathrm{GeV}, m_{v 4}=60 \mathrm{GeV}, m_{\ell 4}=120 \mathrm{GeV}$ and vary $m_{u 4}=m_{d 4}$ between $300 \mathrm{GeV}$ and $650 \mathrm{GeV}$. Such a benchmark case is itself plausible without mixing, particularly when $m_{\ell 4}-m_{v 4}$ mass difference is large. Therefore the favored values of the mixings come out lower than the values in the degenerate-generation case. However, the ratio of the squared mixings, and hence the favored value of $B F$, shows very similar behavior to the degenerate case (Fig. 10, right plot).

In conclusion, the fully degenerate case with mixings is checked to be allowed by the EW precision data. The favored values of the mixing angles $\theta_{24}$ and $\theta_{34}$ tend to imply non-unity branching fractions for the decays of the fourth generation quarks into the third generation final states. The favored value of the $B F$ decreases from about $90 \%$ down to about $60 \%$ with the fourth generation mass increasing from $300 \mathrm{GeV}$ to $650 \mathrm{GeV}$. Light Higgs scenarios with the Higgs boson decaying to 4th generation neutrinos are allowed by the EW precision data. Since mixing increases the $T$ parameter, for heavy charged leptons low values of the mixing would be preferred. However mixing can still play a role with behavior similar to the fully-degenerate case. 
Fig. 8 2D histograms of $\left|\sin \theta_{24}\right|$ and $\left|\sin \theta_{34}\right|$, obtained by using $\chi^{2}$ probability in the $S-T$ space as weights, for three different values of the degenerate fourth-generation fermion mass. (From left to right: $m_{4 \mathrm{G}}=300 \mathrm{GeV}$, $m_{4 \mathrm{G}}=400 \mathrm{GeV}$, $m_{4 \mathrm{G}}=500 \mathrm{GeV}$.) The small black stars at the center of the colored contours indicate the most favored $\left|\sin \theta_{24}\right|-\left|\sin \theta_{34}\right|$ pairs. The scale of the color-axis and the total integral of the weights are normalized to $10^{3}$ non-negative $\left(K_{24}, K_{34}\right)$ pairs (Color figure online)

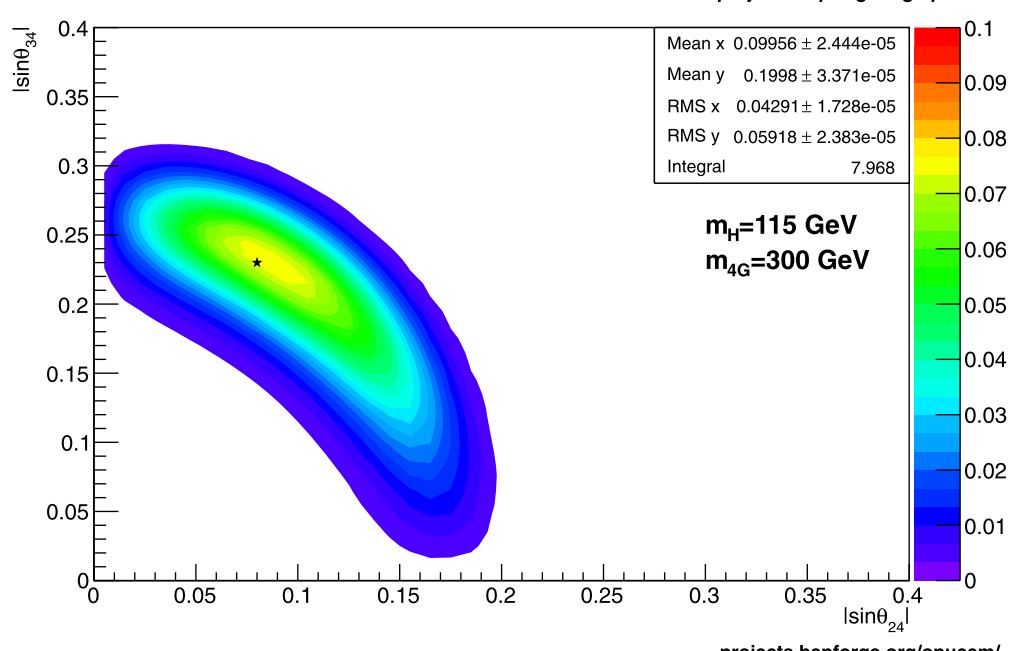

projects.hepforge.org/opucem/

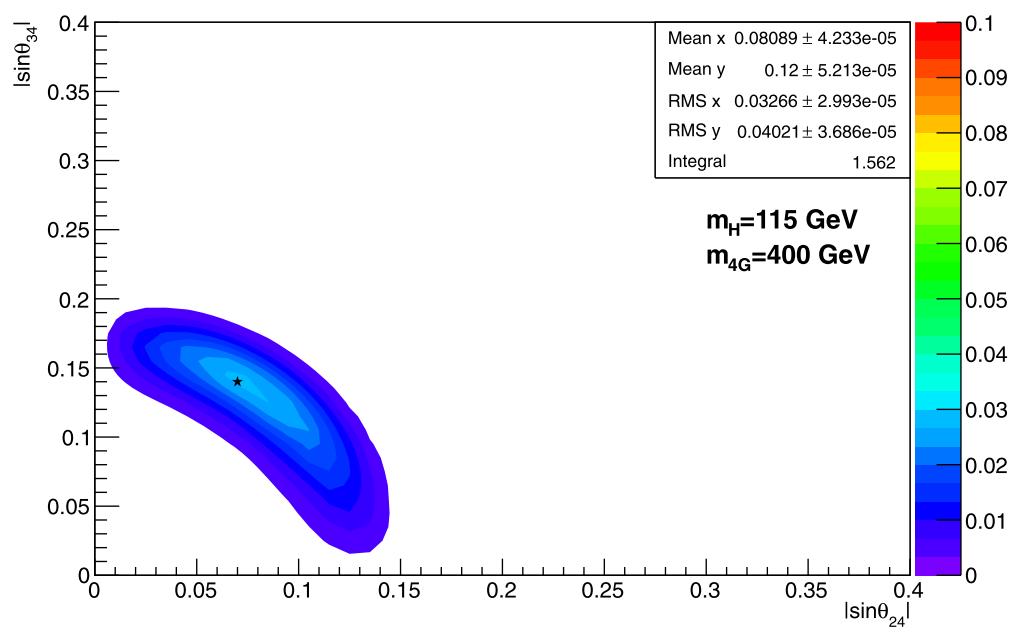

projects.hepforge.org/opucem/

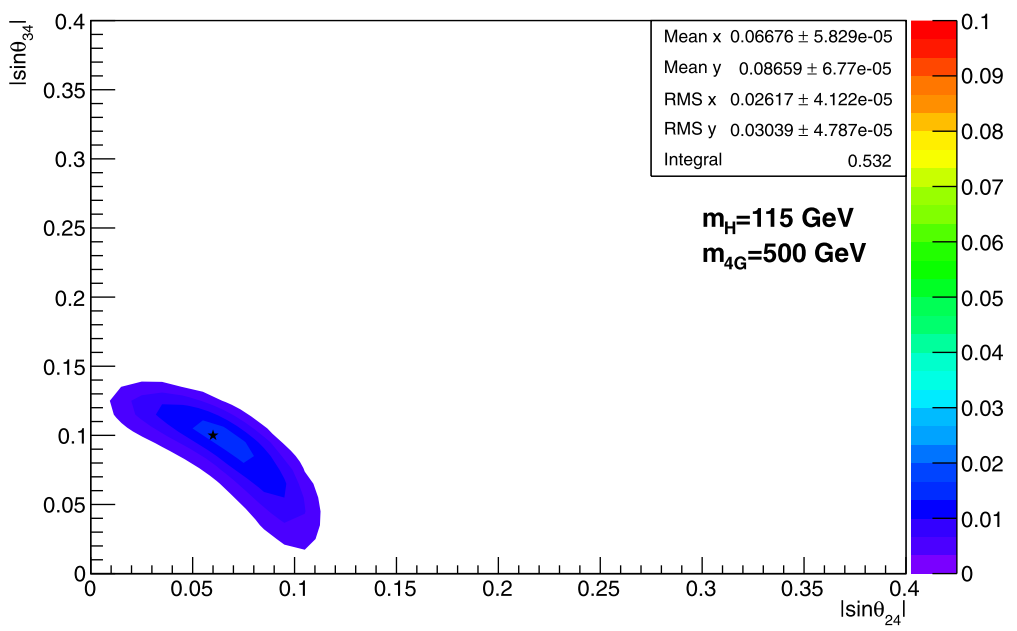

\section{On the fifth and sixth generations}

In this section, OPUCEM has been applied to the Standard Model with 5 and 6 generations (SM5 and SM6) in order to show that these are not excluded by electroweak precision data. It is clear that the lack of a Higgs signal from the LHC so far implies even stronger indirect limits on a simple extension of the Standard Model with 5 or more generations, and might have already excluded some parts of the available parameter space. On the other hand, in certain region of the 

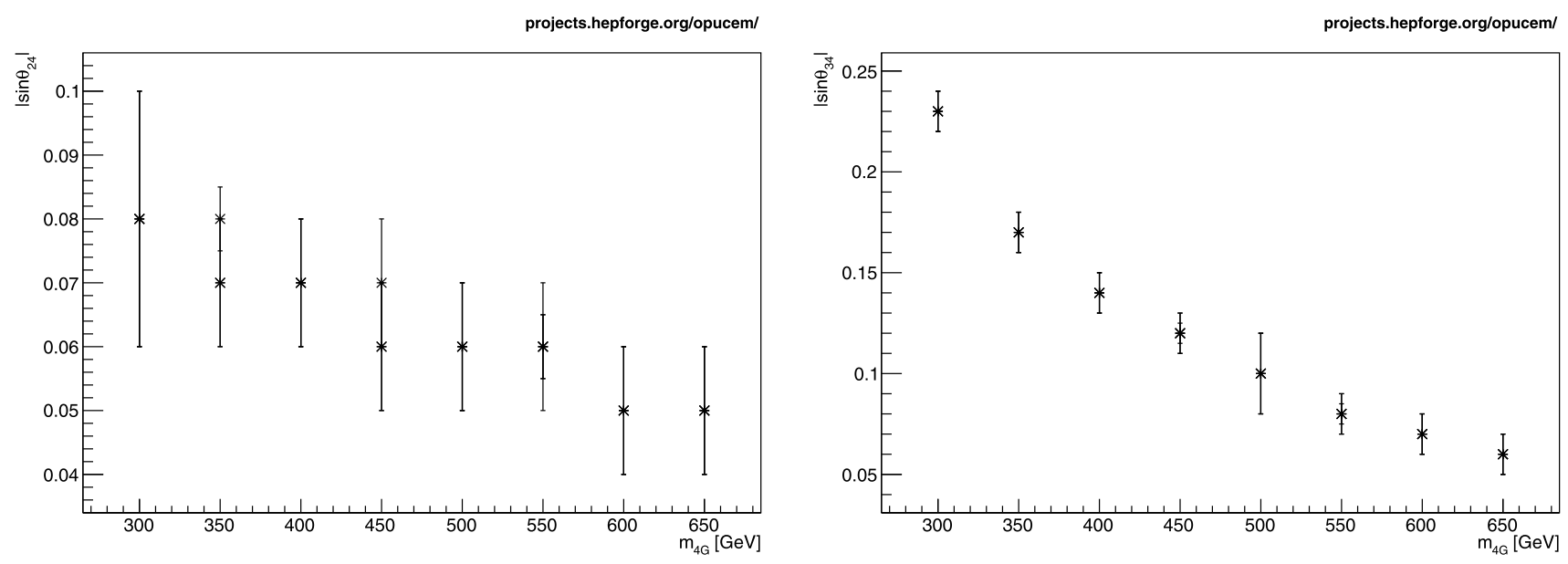

Fig. 9 Favored values of $\left|\sin \theta_{24}\right|$ (left) and $\left|\sin \theta_{34}\right|$ (right) as a function of fourth generation degenerate mass

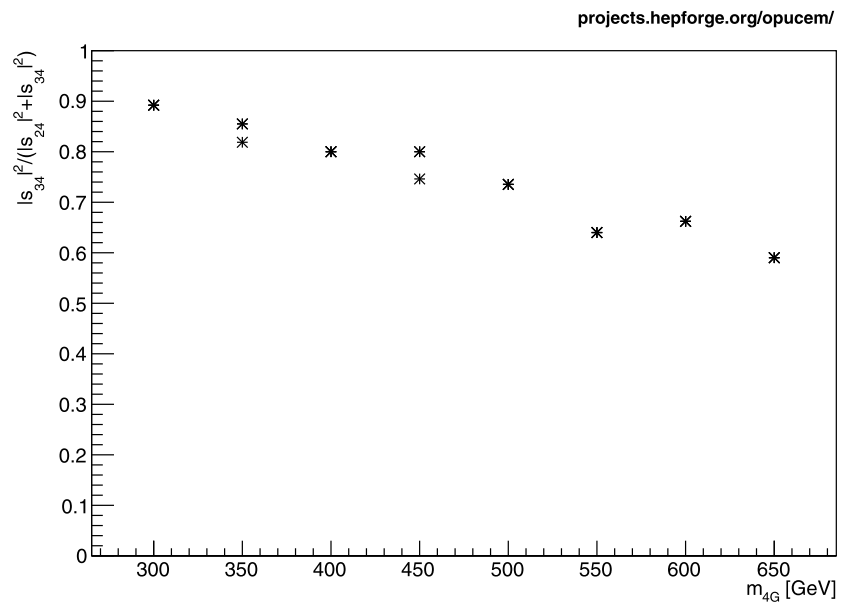

Fig. 10 Left: favored value of $B F \sim\left|V_{t b^{\prime}}\right|^{2}\left(\left|V_{t b^{\prime}}\right|^{2}+\left|V_{c b^{\prime}}\right|^{2}\right)$ as a function of the fourth generation degenerate mass. This plot has been prepared a number of times by iterating the full procedure outlined in the text. For a couple of the $m_{4 \mathrm{G}}$ values, we extract slightly different $B F$

parameter space, the addition of a 5th and 6th generation would reduce the effective cross section in come channels even below the SM3 values [22]. Therefore, a study of these extra-generation cases is still interesting, both as an input to beyond-SM model building with extra generations, and as an exercise in understanding what the EW data might have indicated had it been taken into account appropriately before. Keeping in mind Autumn 2011 LHC results for Higgs boson searches, two values of Higgs boson masses have been considered, namely $m_{H}=115 \mathrm{GeV}$ and $m_{H}=600 \mathrm{GeV}$. The masses of the extra fermions in SM5 with Dirac neutrinos are presented in Table 2 for two example benchmark points. The corresponding points in the $S-T$ plane are shown in Fig. 11 left side together with the SM3 point. It can be seen that all three points are inside the $1 \sigma$ error ellipse. For SM5 with Majorana type neutrinos, two example benchmark

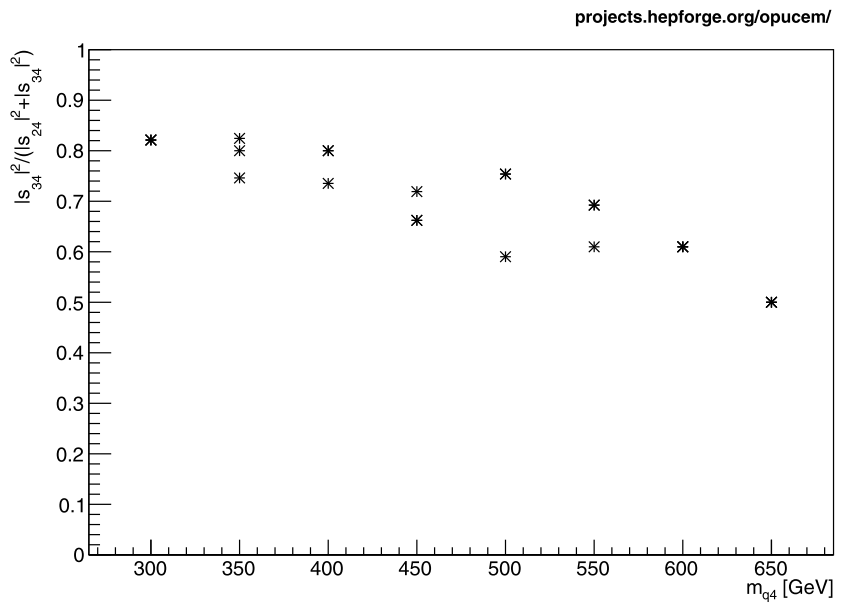

values from these iterations. As a measure of the size of the systematic effects in our procedure, we plot all such values. Right: same plot for the case of a non-degenerate fourth generation scenario with only degenerate quark masses $\left(m_{q 4}=m_{u 4}=m_{d 4}\right)$

Table 2 Two SM5 benchmark points with Dirac type neutrinos. The first point with $m_{H}=115 \mathrm{GeV}$ and $\left|\sin \theta_{34}\right|=0.02$, leading to $S=$ 0.17 and $T=0.17$ is on the left and the second point with $m_{H}=$ $600 \mathrm{GeV}$ and $\left|\sin \theta_{34}\right|=0.02$, leading to $S=0.22$ and $T=0.22$ is on the right

\begin{tabular}{lcccc}
\hline & 4th family & 5th family & 4th family & 5th family \\
\hline$m_{U}(\mathrm{GeV})$ & 500 & 550 & 500 & 550 \\
$m_{D}(\mathrm{GeV})$ & 500 & 550 & 500 & 550 \\
$m_{v}(\mathrm{GeV})$ & 50 & 50 & 50 & 50 \\
$m_{E}(\mathrm{GeV})$ & 120 & 120 & 150 & 165 \\
\hline
\end{tabular}

points are listed in Table 3, with their locations on the $S-T$ plane shown on the right-hand side of Fig. 11.

The SM6 with both Dirac and Majorana neutrinos has also been investigated. Table 4 contains two example sets of 


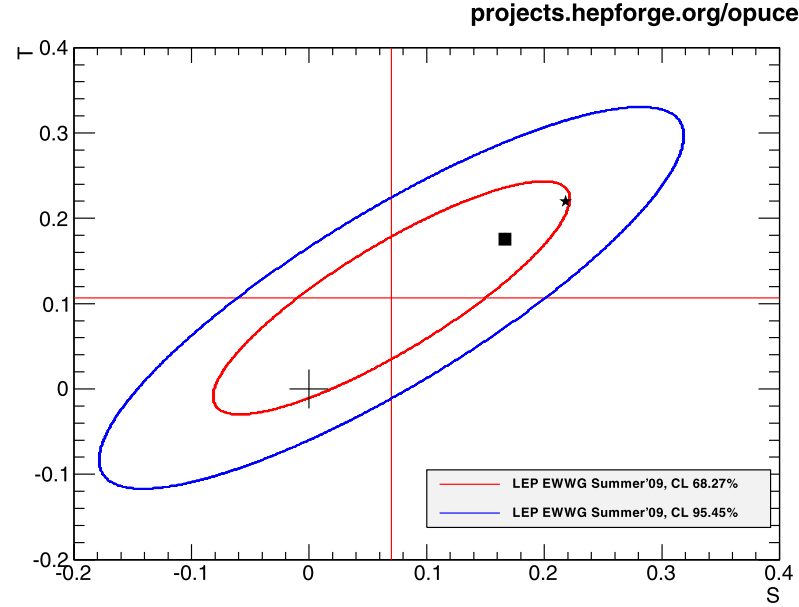

Fig. 11 SM3 and four SM5 benchmark points in $S-T$ plane with Dirac (left) or Majorana (right) type neutrinos. The $1 \sigma$ and $2 \sigma$ error ellipses represent the 2009 results of the $U=0$ fit from the LEP EWWG. The cross corresponds to the SM3 with $m_{H}=115 \mathrm{GeV}$. In

Table 3 Two SM5 benchmark points with Majorana type neutrinos. First point with $m_{H}=115 \mathrm{GeV}$ and $\left|\sin \theta_{34}\right|=0.07$, leading to $S=$ 0.11 and $T=0.13$ is on the left and second point with $m_{H}=600 \mathrm{GeV}$ and $\left|\sin \theta_{34}\right|=0.07$, leading to $S=0.22$ and $T=0.22$ is on the right

\begin{tabular}{lcccc}
\hline & 4th family & 5th family & 4th family & 5th family \\
\hline$m_{U}(\mathrm{GeV})$ & 570 & 580 & 590 & 590 \\
$m_{D}(\mathrm{GeV})$ & 500 & 500 & 500 & 500 \\
$m_{v}(\mathrm{GeV})$ & 260 & 45 & 260 & 45 \\
$m_{E}(\mathrm{GeV})$ & 590 & 510 & 590 & 510 \\
$m_{N}(\mathrm{GeV})$ & 2550 & 2900 & 2550 & 2900 \\
\hline
\end{tabular}

Table 4 Two SM6 benchmark points with Dirac type neutrinos. The first point with $m_{H}=115 \mathrm{GeV}$ and $\left|\sin \theta_{34}\right|=0.00$, leading to $S=$ 0.25 and $T=0.26$ is on the left and the second point with $m_{H}=$ $600 \mathrm{GeV}$ and $\left|\sin \theta_{34}\right|=0.01$, leading to $S=0.31$ and $T=0.31$ is on the right

\begin{tabular}{lrrrrrr}
\hline & $\begin{array}{l}\text { 4th } \\
\text { family }\end{array}$ & $\begin{array}{l}\text { 5th } \\
\text { family }\end{array}$ & $\begin{array}{l}\text { 6th } \\
\text { family }\end{array}$ & $\begin{array}{l}\text { 4th } \\
\text { family }\end{array}$ & $\begin{array}{l}\text { 5th } \\
\text { family }\end{array}$ & $\begin{array}{l}\text { 6th } \\
\text { family }\end{array}$ \\
\hline$m_{U}(\mathrm{GeV})$ & 530 & 550 & 580 & 580 & 580 & 590 \\
$m_{D}(\mathrm{GeV})$ & 520 & 550 & 580 & 580 & 580 & 590 \\
$m_{v}(\mathrm{GeV})$ & 50 & 50 & 50 & 50 & 50 & 50 \\
$m_{E}(\mathrm{GeV})$ & 110 & 130 & 120 & 110 & 140 & 180 \\
\hline
\end{tabular}

mass values for the additional generations with Dirac type neutrinos. Corresponding points in $S-T$ plane are shown in Fig. 12 left side. It is seen that although the SM6 points lie further away from the center of the ellipse than the SM3 point, they are still within $2 \sigma$ error ellipse, thus not ruled out by the EW data. Similarly, two example sets of mass values for SM6 with Majorana type neutrinos are presented in Table 5 and the corresponding points in $S-T$ plane are shown in Fig. 12 right side. One can see that these points

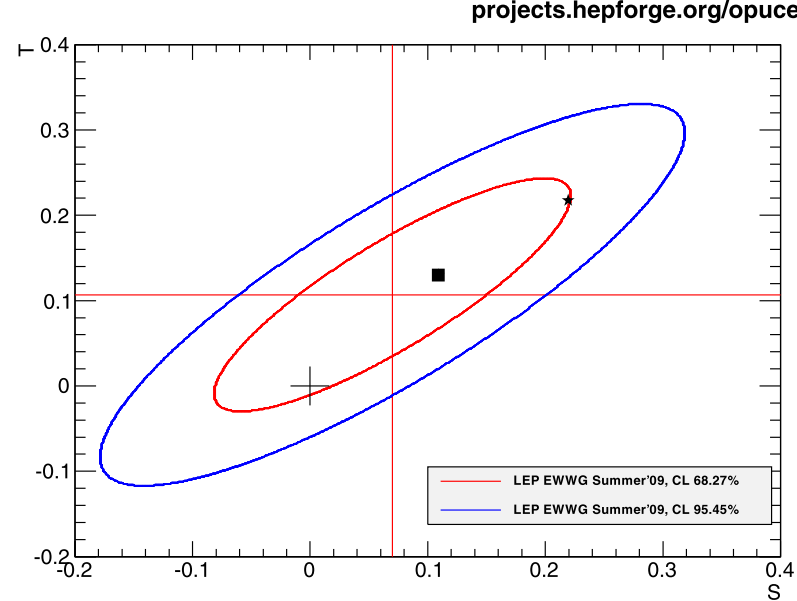

the left plot, the square (star) corresponds to the first (second) SM5 point in Table 2. In the right plot, the square (star) corresponds to the first (second) SM5 point in Table 3

Table 5 Two SM6 benchmark points with Majorana type neutrinos. The first point with $m_{H}=115 \mathrm{GeV}$ and $\left|\sin \theta_{34}\right|=0.01$, leading to $S=0.09$ and $T=0.12$ is on the left and the second point with $m_{H}=$ $600 \mathrm{GeV}$ and $|\sin \theta|=0.05$, leading to $S=0.20$ and $T=0.21$ is on the right

\begin{tabular}{lrrrrrr}
\hline & $\begin{array}{l}\text { 4th } \\
\text { family }\end{array}$ & $\begin{array}{l}\text { 5th } \\
\text { family }\end{array}$ & $\begin{array}{l}\text { 6th } \\
\text { family }\end{array}$ & $\begin{array}{l}\text { 4th } \\
\text { family }\end{array}$ & $\begin{array}{l}\text { 5th } \\
\text { family }\end{array}$ & $\begin{array}{l}\text { 6th } \\
\text { family }\end{array}$ \\
\hline$m_{U}(\mathrm{GeV})$ & 560 & 570 & 570 & 580 & 580 & 580 \\
$m_{D}(\mathrm{GeV})$ & 500 & 500 & 500 & 500 & 500 & 510 \\
$m_{\nu}(\mathrm{GeV})$ & 45 & 45 & 260 & 45 & 45 & 260 \\
$m_{E}(\mathrm{GeV})$ & 410 & 480 & 550 & 410 & 480 & 550 \\
$m_{N}(\mathrm{GeV})$ & 2400 & 2600 & 3500 & 2200 & 2500 & 3500 \\
\hline
\end{tabular}

are within $1 \sigma$ error ellipse. It is worth noting that the SM6 points with $m_{H}=115 \mathrm{GeV}$ is significantly closer to central value than the SM3 point.

\section{Conclusions}

Using the enhancements to the OPUCEM library and associated tools, it is shown that given the Autumn 2011 direct search results from the LHC experiments, a sequential fourth family is still consistent with the electroweak precision data. The statement is valid for both cases with Dirac and Majorana neutrinos and also for both light and heavy Higgs bosons. It should also be noted that the EW data still prefers a light Higgs for both Dirac and Majorana type fourth generation. Consideration of the mixing with light generation quarks enlarges the available parameter space, making even the fully degenerate mass scenario compatible with the EW precision data. Such a mixing also reduces the current SM4 quark mass limits, as the favored $B F$ to the 


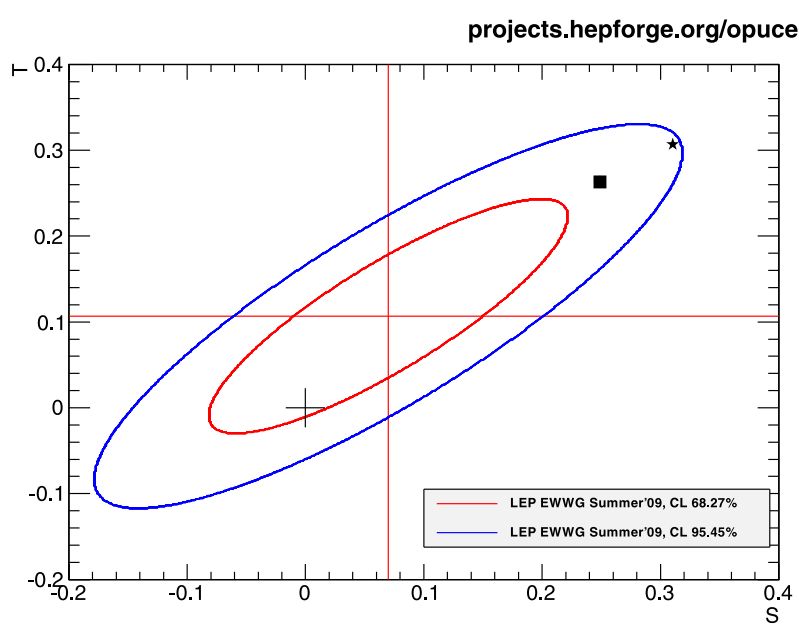

Fig. 12 SM3 and four SM6 benchmark points with Dirac (left) and Majorana (right) type neutrinos. The $1 \sigma$ and $2 \sigma$ error ellipses represent the 2009 results of the $U=0$ fit from the LEP EWWG. The cross corresponds to the SM3 with $m_{h}=115 \mathrm{GeV}$. In the left plot, the square

third generation is found to be less than 100 percent. Finally, analysis with OPUCEM shows that a fifth or even a sixth sequential generation has been in the realm of the possible, with a $\chi^{2}$ lower than the three-generation SM in some example benchmark cases. In conclusion, it is worth highlighting that the only way to discover or completely rule out models with additional generations relies on further data collection at the LHC and its analysis.

Acknowledgements We would like to thank Saleh Sultansoy for many helpful conversations and discussions. M. Şahin's work is supported by TUBITAK BIDEB-2218 grant. G. Unel's work is supported in part by U.S. Department of Energy Grant DE FG0291ER40679. The collaboration leading to this paper has partially been possible thanks to funds provided by the Boğaziçi University Foundation.

Open Access This article is distributed under the terms of the Creative Commons Attribution License which permits any use, distribution, and reproduction in any medium, provided the original author(s) and the source are credited.

\section{References}

1. D.C. Kennedy, B.W. Lynn, Electroweak radiative corrections with an effective Lagrangian: four-fermions processes. Nucl. Phys. B 322, 1-54 (1989)

2. M.E. Peskin, T. Takeuchi, Estimation of oblique electroweak corrections. Phys. Rev. D 46, 381 (1992)

3. OPUCEM project at CEDAR Hepforge repository, http://projects. hepforge.org/opucem/

4. B. Holdom et al., Four statements about the fourth generation. PMC Phys. A 3, 4 (2009)

5. Ö. Çobanoglu et al., OPUCEM: a library with error checking mechanism for computing oblique parameters. Comput. Phys. Commun. 182, 1732 (2011)

6. V.E. Özcan (on behalf of the ATLAS and CMS Collaborations), Searches for exotic heavy quarks at the LHC. Presented at TOP 2011, 4th International Workshop on Top Quark Physics (2011) projects.hepforge.org/opucem/

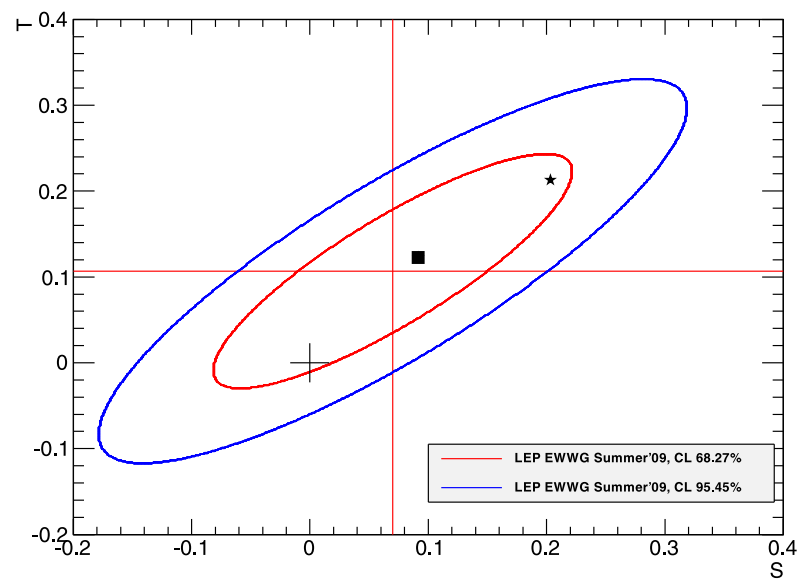

(star) corresponds to first (second) SM6 point in Table 4. In the right plot, the square (star) corresponds to first (second) SM6 point in Table 5

7. M. Baak et al. (The Gfitter Group), Updated status of the global electroweak fit and constraints on new physics. Eur. Phys. J. C (submitted). arXiv:1107.0975 [hep-ph]

8. T. Plehn et al., G4LHC-four generations at LHC. http://www. thphys.uni-heidelberg.de/ plehn/index.php?show=fourth_gen\& visible $=$ tools

9. G. Kribs et al., Four generations and Higgs physics. Phys. Rev. D 76, 075016 (2007). doi:10.1103/PhysRevD.76.075016. arXiv: 0706.3718 [hep-ph]

10. Tevatron Electroweak Working Group, Combination of CDF and D0 results on the mass of the top quark. arXiv:0903.2503 [hep-ex]. FERMILAB-Tm-2427-E

11. C. Amsler et al. (Particle Data Group), Review of particle physics. Phys. Lett. B 667, 1 (2008)

12. K.-F. Chen, Direct searches for 4 th generation quarks at CMS. Presented at Flavour and fourth family workshop, Durham, UK, 14 September 2011

13. CMS Collaboration, Search for a heavy bottom-like quark in $1.14 \mathrm{fb}^{-1}$ of pp collisions at $\sqrt{s}=7 \mathrm{TeV}$. CMS PAS EXO-11$036(2011)$

14. CMS Collaboration, Search for pair production of a fourthgeneration $t^{\prime}$ quark in the lepton-plus-jets channel with the CMS experiment. CMS PAS EXO-11-051 (2011)

15. CMS Collaboration, SM Higgs combination. CMS-PAS-HIG-11$011(2011)$

16. ATLAS Collaboration, Update of the combination of Higgs boson searches in $p p$ collisions at $\operatorname{sqrt}(s)=7 \mathrm{TeV}$ with the ATLAS experiment at the LHC. ATLAS-CONF-2011-135 (2011)

17. S.P. Martin, Extra vector-like matter and the lightest Higgs scalar boson mass in low-energy supersymmetry. Phys. Rev. D 81, 035004 (2010). arXiv:0910.2732v2 [hep-ph]

18. O. Eberhardt, A. Lenz, J. Rohrwild, Less space for a new family of fermions. Phys. Rev. D 82, 095006 (2010). arXiv: 1005.3505

19. A. Lenz, B-physics and EW constraints on SM4. Presented at B3SM-III, third workshop on beyond 3 generation standard model, Istanbul (2011)

20. ATLAS and CMS Collaborations, Combined standard model Higgs boson searches with up to $2.3 \mathrm{fb}^{-1}$ of pp collisions at $\sqrt{s}=7 \mathrm{TeV}$ at the LHC. ATLAS-CONF-2011-157, CMS-PASHIG-11-023 
21. S.A. Cetin, T. Çuhadar-Dönszelmann, M. Sahin, S. Sultansoy, G. Unel, Impact of the relatively light fourth family neutrino on the Higgs boson search. Phys. Lett. B (accepted for publication). arXiv:1108.4071
22. E. Arik, O. Cakir, S.A. Cetin, S. Sultansoy, Observability of the Higgs boson in the presence of extra standard model families at the Tevatron. Acta Phys. Pol. B 37 (2006). arXiv:hep-ph/ 0502050 